\title{
Ensuring Racial Equity in Midwifery Education Programs: Beginning Steps for Midwifery Education.
}

\author{
Ronnie Lichtman \\ SUNY Downstate Health Sciences University \\ Jennifer Woo \\ Texas Woman's University \\ Pamela Reis \\ East Carolina University \\ Carrie S. Klima \\ University of Illinois Chicago \\ Dana Perlman \\ Thomas Jefferson University, dana.perlman@jefferson.edu \\ Follow this and additional works at:https://jđc.jefferson.edu/jchpfp \\ Part of the Nursing Midwifery Commons \\ Let us know how access to this document benefits you
}

\section{Recommended Citation}

Lichtman, Ronnie; Woo, Jennifer; Reis, Pamela; Klima, Carrie S.; and Perlman, Dana, "Ensuring Racial Equity in Midwifery Education Programs: Beginning Steps for Midwifery Education." (2020). College of Health Professions Faculty Papers. Paper 3.

https://jdc.jefferson.edu/jchpfp/3

This Article is brought to you for free and open access by the Jefferson Digital Commons. The Jefferson Digital Commons is a service of Thomas Jefferson University's Center for Teaching and Learning (CTL). The Commons is a showcase for Jefferson books and journals, peer-reviewed scholarly publications, unique historical collections from the University archives, and teaching tools. The Jefferson Digital Commons allows researchers and interested readers anywhere in the world to learn about and keep up to date with Jefferson scholarship. This article has been accepted for inclusion in College of Health Professions Faculty Papers by an authorized administrator of the Jefferson Digital Commons. For more information, please contact: JeffersonDigitalCommons@jefferson.edu. 


\section{ENSURING RACIAL EQUITY IN MIDWIFERY EDUCATION PROGRAMS: BEGINNING STEPS FOR MIDWIFERY EDUCATION}

\section{INTRODUCTION}

In a 1981 editorial in the Journal of Nurse-Midwifery, Betty Carrington called for the recruitment of more individuals of color into the midwifery profession ${ }^{1}$. Citing descriptive research that concluded that the percentage of Blacks and persons of Hispanic ethnicity in nurse-midwifery was lower than that of the general population, she wrote "let us make a greater effort to see that it (midwifery) represents the cultural and ethnic richness of American society." " (p2) Unfortunately, significant progress has not been made in reaching the goals set out by this early midwifery leader regarding racial and ethnic diversity. ${ }^{2}$ More than 25 years later, an analysis of the membership data of the American College of Nurse-Midwives (ACNM) showed that only 7\% of ACNM members and $5.8 \%$ of midwives recertifying through the American Midwifery Certification Board (AMCB) were identified as people of color, although $14.5 \%$ of midwives initially certified by AMCB were identified as people of color $^{2}$. A review of the literature conducted in 2016, 35 years after Carrington's call for action, concluded that even with this increased number of persons of color entering into midwifery, the largest group of midwives remains white, at a higher percentage than makes up the general population of the United States. ${ }^{3}$

Today, in light of the ever-increasing awareness of the need to create a midwifery workforce that reflects the people we serve and the changing demographics of our nation ${ }^{4}$, addressing issues of diversity and inclusion within our education programs has become imperative. Taking a critical look at these issues is even more important in light of recent national exposure through social media of violence against Black, Indigenous, 
and People of Color in the United States; the renewed and vocal outcry against such violence $^{5}$; the recognition of the startling and unacceptable inequities in maternal and newborn morbidity and mortality ${ }^{6}$, and the inexcusable world standing of the United States in terms of these issues ${ }^{7}$. An evaluation of diversity and its various components can provide background and support for current and future initiatives within the midwifery profession and its educational programs around racial injustice and the need for diversity, equity, and inclusion.

The purpose of this commentary is to describe a beginning step that the Directors of Midwifery Education (DOME) took toward ensuring racial equity in midwifery education programs: the gathering of data. We will discuss barriers identified in the most basic components of ensuring equity and advocate for a concerted effort among stakeholders in midwifery education, DOME, ACNM, the Accreditation Commission for Midwifery Education (ACME), and AMCB, to address critical barriers.

\section{AN INITIATIVE FROM DIRECTORS OF MIDWIFERY EDUCATION}

DOME is a comprised of the program directors of all ACME-accredited midwifery education programs. Its broad purpose is to advance midwifery education through sharing of information, mentoring of new directors, and addressing issues relevant to midwifery education. The DOME members have the unique role of representing educators who prepare the next generation of midwives. In recognition of the importance and challenge of increasing the diversity within our profession, in 2016 DOME convened a Diversity and Inclusion Task Force consisting of volunteers from the organization. Although the task force was not given a mission by DOME, its members made the decision to start its work by collecting data from program directors and chairs 
on diversity among students and faculty within every midwifery program. What we discovered was that this was a difficult and complicated task due to the lack of a standardized methodology or tool for such data collection or reporting among midwifery education programs.

\section{Our Task: Gathering Data}

The task force designed a survey with input from the Midwives of Color Committee Chair. The survey included closed-ended questions about diversity and inclusion and open-ended questions about access to diversity and inclusion resources within the program and institution, including activities that the program had undertaken that were beneficial. Questions about diversity and inclusion included those relating to race, ethnicity, gender, disability, first generation college student, sexual orientation, and gender identity. Sexual orientation and gender identity were included together in the category LGBTQIA (lesbian, gay, bisexual, transgender, queer, intersex, asexual). Various aspects of student and faculty diversity and access to diversity and inclusion resources were also examined. The survey and study design were approved by the Institutional Review Boards of Thomas Jefferson University and SUNY Downstate Health Sciences University.

\section{What We Learned}

Twenty-eight program directors or chairs responded to the DOME survey. Their responses represent $72 \%$ of all United States (US) midwifery education programs and 131 faculty and 549 students across these programs. While most programs reported that they collected data on the race and ethnicity of students, there were a small number of programs who either did not collect data on race and ethnicity, or whose data were incomplete. Of the total number of students of color for whom race and ethnicity were 
reported, $14 \%$ were Black/African American, $3.6 \%$ were Asian, $2.9 \%$ were Latinx, $0.7 \%$ were Native American/Alaska Native, and $0.2 \%$ were Native Hawaiian/Other Pacific Islander. Approximately 8\% of students identified as multiracial. Only 3 students who identified as male $(0.5 \%)$ were reported; the race and ethnicity of the male students were not captured.

The range of faculty of color per program was $0-4$. Among the midwifery program core faculty for whom race and ethnicity were reported, $9.2 \%$ were Black/African American, 3.8\% were Latinx, 3.0\% were Asian, $3.0 \%$ were multiracial, and $1.5 \%$ were Native American/Alaska Native. None of the faculty reported in the survey identified as Native Hawaiian/Other Pacific Islander.

Most programs reported having resources to serve the needs of underrepresented students at the university or academic institution (86\%), specific college, school, or department $(71.4 \%)$, or midwifery program $(60.7 \%)$. These resources included required course content that addressed issues of race, racism, diversity, and inclusion, and resources for development of content about race, racism, diversity, and inclusion through their academic institution, including professional development, special curricula and programming, and multicultural centers. Some examples of the activities implemented or sponsored specifically by midwifery education programs are an online multicultural center, a review of midwifery historical literature to understand the role and challenges of African American midwives in the early $20^{\text {th }}$ century, creation of a Chief Diversity and Inclusion Officer position, holistic admission processes, required content within the curriculum that includes presentations by midwives of color, and workshops on transgender and queer care. 


\section{Where We Need to Improve}

Unfortunately, one striking observation that came from this effort was the lack of standardization of record keeping related to student and faculty diversity across programs. For example, there did not appear to be consistency in the extent of diversity data collected by the programs. Some look at first generation college attendance; others do not. Also, we were unable to discern how programs collected information on student or faculty diversity. Was it collected by the school, college, or university or the program itself? If the former, might statistics reported be combined with those of other programs in the same unit? Clearly, in order to assess improvements or lack of progress in student body diversity, and to develop and implement initiatives to diversify the midwifery workforce through increased enrollments of students from underrepresented groups, midwifery programs must take steps to ensure that data collected are consistent about various aspects of student diversity.

A second observation is that while the majority of programs collected data about racial and ethnic diversity, most programs did not collect student or faculty data about other aspects of diversity, such as LGBTQIA identity, disability status, students or faculty who identified as first-generation college students, or if English was a second language for students or faculty. For example, there was a lack of formalized mechanisms to keep track of midwifery students who identify as first-generation college students, despite research that shows that such students fall into a vulnerable category. ${ }^{8}$ Further discussion of diversity should also include the broad areas of gender identity and sexual orientation. While all programs reported that they collected information on student sex using simple binary female/male designations, only $25 \%$ of midwifery educational programs collect information about sexual orientation, gender identity, or 
gender expression of their students or faculty. The number of midwifery students who may identify not only as a racial or ethnic minority, but also as LGBTQIA is unknown, as is the number of faculty who so identify.

There was also a notable lack of diversity of current faculty among the 28 programs that responded to the survey. For example, $14.2 \%$ of the student body reported in the data are of Non-Hispanic Black ethnicity yet only $9.1 \%$ of the faculty currently teaching in midwifery programs are Non-Hispanic Black. In order to increase diversity of students entering midwifery programs, greater effort to diversify midwifery faculty is needed. Several studies in the health sciences support this contention. In a survey of recruitment strategies among physician assistant programs in the United States, one of the correlates of self-reported success in recruitment of a more diverse student body was a greater proportion of African American and Hispanic faculty on admissions committees. ${ }^{9}$ A qualitative study about diversity in nursing education found that faculty diversity was one of several factors that influenced student diversity. ${ }^{10}$ Another qualitative study of five medical schools concluded that "achieving an inclusive culture for diverse medical school faculty would help meet the mission of academic medicine to train a physician and research workforce."11 (p1363)

Finally, additional data are needed about how diversity services are implemented and utilized by midwifery programs and students. According to the current survey results, $75 \%$ of programs have both an office and officer of diversity and inclusion and $60.7 \%$ have support services specifically for underrepresented students, yet it is not clear what impact these services have in increasing diversity at a macro level or improving students' 
experiences on a micro level. In fact, our survey did not capture if and how these services are utilized at all.

\section{RECOMMENDATIONS AND CONCLUSION}

This effort by the DOME Diversity and Inclusion Task Force has identified significant gaps in how certain variables related to diversity are defined and collected by midwifery education programs nationwide. As a result, the DOME task force calls for a collaborative effort among DOME, ACNM, ACME, and AMCB to develop clearer and more robust standardized definitions of various components of diversity in midwifery education. We also call for a consistent methodology for data collection by midwifery programs themselves, as opposed to relying on college or university level data. We advocate for full transparency in reporting of data within midwifery programs similar to the way universities report these data for prospective applicants. We support the setting of benchmarks for these metrics, not in terms of specific numbers, but in terms of improvement over time. Openness and transparency among the stakeholders will allow for accountability in enrollment, accreditation, and certification, which is the only way to ensure success in increasing the diversity of midwifery education programs and thus the midwifery workforce. It is also a huge step toward ensuring equity.

One of the challenges will be to address and examine the specific needs of students who are typically underrepresented in midwifery programs. ${ }^{10}$ A vital step to further elucidating the experience and needs of underrepresented students in midwifery programs is to survey the students themselves, particularly with regard to how they are currently being supported and in what ways they are not.

Additional recommendations for furthering the important work of diversifying the midwifery workforce must acknowledge institutional racism and how midwifery 
programs could be perpetuating a culture of whiteness at the expense of midwifery students of color. This will require a multi-pronged research and action approach that will determine appropriate metrics to measure and assess the ways midwifery programs recruit students of color; to analyze how white supremacy and racism affect all aspects of the experiences of students of color in midwifery programs, and perhaps even discourages students from pursuing midwifery as a profession; to acknowledge and recognize the accomplishments of midwives of color; and to develop ongoing and indepth anti-racist curricula and training for both faculty and students. Such work has been long overdue within the profession of midwifery. Now is the time for midwifery to take a stance and be proactive in diversifying the midwifery workforce, starting with midwifery education, for the betterment of mothers and babies in this country. 


\section{References}

1. Carrington B. Minority recruitment. J Nurse Midwifery. 1981; 26(1):1-2. doi:10.1016/00912182(81)90198

2. Fullerton J, Sipe TA, Hastings-Tolsma M, et al. The midwifery workforce: ACNM 2012 and AMCB 2013 core data. J Midwifery Womens Health. 2015; 60(6):751-761. doi:10.1111/jmwh.12405

3. Wren Serbin J, Donnelly E. The Impact of Racism and Midwifery's Lack of Racial Diversity: A Literature Review. J Midwifery Womens Health. 2016; 61(6):694-706. doi:10.1111/jmwh.12572

4. U.S. Census Bureau projections show a slower growing, older, more diverse nation a half century from now. U.S. Census Bureau website. https://www.census.gov/newsroom/releases/archives/population/cb12-243.html. Published December 12, 2012. Accessed September 21, 2020.

5. Sobo EJ, Lambert H, Heath CD. More than a teachable moment: Black lives matter. Anthropol Med. 2020; 27(3):243-248. doi:10.1080/13648470.2020.1783054

6. Infographic: Racial/Ethnic Disparities in Pregnancy-Related Deaths — United States, 20072016. Centers for Disease Control and Prevention. https://www.cdc.gov/reproductivehealth/maternal-mortality/disparities-pregnancy-relateddeaths/infographic.html. Accessed September 21, 2020.

7. GBD 2015 Maternal Mortality Collaborators. Global, regional, and national levels of maternal mortality, 1990-2015: A systematic analysis for the Global Burden of Disease Study 2015. The Lancet. 2016; 388(10053. doi: https://doi.org/10.1016/S0140.6736(16)31470-2

8. Ward L, Siegel MJ, Davenport Z. First-Generation College Students: Understanding and Improving the Experience from Recruitment to Commencement. John Wiley \& Sons; 2012.

9. DiBaise M, Salisbury H, Hertelendy A, Muma RD. Strategies and perceived barriers to recruitment of underrepresented minority students in physician assistant programs. $J$ Physician Assist Educ. 2015; 26(1):19-27. doi:10.1097/JPA.0000000000000005

10. Gates SA. What works in promoting and maintaining diversity in nursing programs. Nurs Forum. 2018; 53(2):190-196. doi:10.1111/nuf.12242

11. Pololi L, Cooper LA, Carr P. Race, Disadvantage and Faculty Experiences in Academic Medicine. J Gen Intern Med. 2010; 25(12):1363-1369. doi:10.1007/s11606-010-1478-7 